\title{
On the algebraic approach to solvable lattice models
}

\author{
Vladimir Belavin ${ }^{a, b, c}$ and Doron Gepner ${ }^{a}$ \\ ${ }^{a}$ Department of Particle Physics and Astrophysics, Weizmann Institute, \\ Herzl st. 234, Rehovot 76100, Israel \\ ${ }^{b}$ I.E. Tamm Department of Theoretical Physics, P.N. Lebedev Physical Institute, \\ Leninsky av. 53, 11991 Moscow, Russia \\ ${ }^{c}$ Department of Quantum Physics, Institute for Information Transmission Problems, \\ Bolshoy Karetny per. 19, 127994 Moscow, Russia \\ E-mail: vlbelavin@gmail.com, doron.gepner@weizmann.ac.il
}

ABSTRACT: We treat here interaction round the face (IRF) solvable lattice models. We study the algebraic structures underlining such models. For the three block case, we show that the Yang Baxter equation is obeyed, if and only if, the Birman-Murakami-Wenzl (BMW) algebra is obeyed. We prove this by an algebraic expansion of the Yang Baxter equation (YBE). For four blocks IRF models, we show that the BMW algebra is also obeyed, apart from the skein relation, which is different. This indicates that the BMW algebra is a sub-algebra for all models with three or more blocks. We find additional relations for the four block algebra using the expansion of the YBE. The four blocks result, that is the BMW algebra and the four blocks skein relation, is enough to define new knot invariant, which depends on three arbitrary parameters, important in knot theory.

KEywords: Conformal Field Theory, Integrable Field Theories, Lattice Integrable Models

ARXiv EPRINT: 1810.07959 


\section{Contents}

1 Introduction 1

2 Interaction round the face lattice models $\quad 2$

3 Birman-Murakami-Wenzl algebra 4

$\begin{array}{lll}4 & \text { Four blocks lattice IRF models } & 7\end{array}$

$5 \quad$ The fused $3 \times 3 \mathrm{SU}(2)$ model $\quad 9$

6 Discussion 10

$\begin{array}{ll}\text { A The relations of the three block YBE algebra } & 10\end{array}$

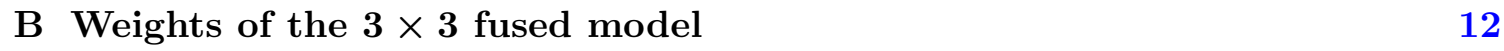

C The algebraic expansion of the four block YBE 14

$\begin{array}{ll}\text { D New knot invariants } & 15\end{array}$

\section{Introduction}

Our interest in this paper is solvable interaction round the face (IRF) lattice models in two dimensions. The solvable lattice models are important playground to study statistical mechanics systems and their phase structure. For a review see [1, 2].

The IRF lattice models are strongly connected with two dimensional conformal field theory. First, the models have a second order phase transition points which are described by some conformal field theory. Second, the construction of the models themselves is achieved through the data of some conformal field theories [3]. Apart from describing second order phase transitions, the conformal field theories are important in string theory compactifications, where they describe the world sheet dynamics, see, e.g., [4].

Our purpose in this paper is to describe the algebraic structure of solvable IRF lattice models. We have already started this investigation in a previous paper [5], where it was argued that any three blocks IRF model obeys the Birman-Murakami-Wenzl (BMW) algebra $[6,7]$. Here, we wish to prove this result in detail along with proving that the Yang Baxter equation (YBE) is obeyed if the BMW algebra holds.

We then study four block theories and we show that they too obey the BMW algebra, with a different skein relation. We exemplify this result by calculating numerically the algebra from the $3 \times 3$ fused $\mathrm{SU}(2)$ model [8]. 


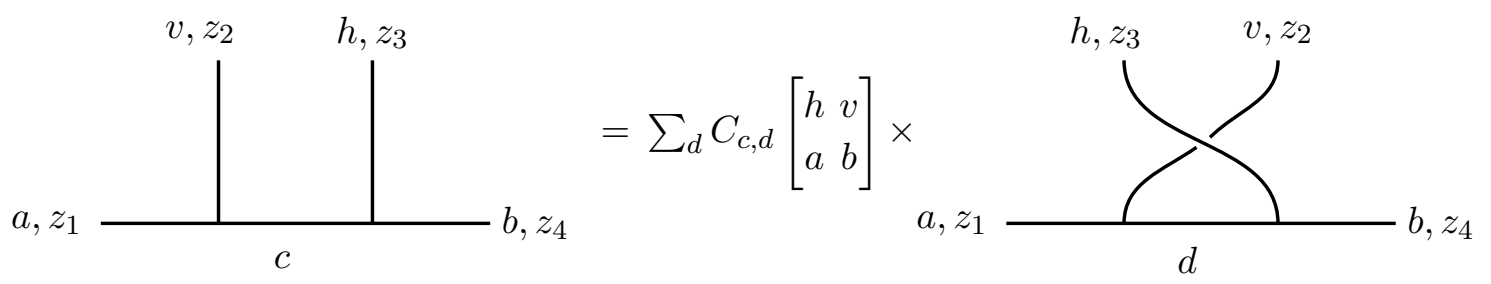

Figure 1. Braiding matrix.

This result is enough to generate all the relations of the four block algebra. It is also sufficient to define a new knot invariant, using the algebraic relations to 'reduce' words in the braid group enveloping algebra. Thus, this algebraic structure is important in knot theory. For a review on the application of IRF models to knot theory, see [2] and also [9].

The connection between solvable lattice models and BMW algebra was discussed previously in connection to the BCD models ref. [2], and refs. therein, for certain superalgebras [10] and in connection to the Izergin-Korepin model [11]. We agree with these works and our results generalize them.

We hope that our results will further the understanding of solvable lattice models. An important question is to figure out the algebra underlying the general $n$ block lattice model, with arbitrary $n$. We conjecture that the BMW algebra (without the skein relation) is a sub-algebra for any number of blocks greater or equal three, $n \geq 3$.

\section{Interaction round the face lattice models}

We define an Interaction Round the Face (IRF) lattice model from the braiding matrix of a rational conformal field theory model. The Boltzmann weights obey the limit

$$
\lim _{u \rightarrow i \infty} g(u) \omega\left(\begin{array}{ll}
a & b \\
c & d
\end{array} \mid u\right)=C_{c, d}\left[\begin{array}{ll}
h & v \\
a & b
\end{array}\right],
$$

where $\omega$ is the Boltzmann weight and $C$ is the Braiding matrix of the conformal field theory [12], i.e., the braiding of the four point conformal blocks (see figure 1), and $g(u$ ) is some irrelevant function, used to make the limit finite. The primary fields $h$ and $v$ are some fixed primary fields used in the definition of the IRF model and $a, b, c, d$ are any of the primary fields of the conformal field theory, $\mathcal{O}$. The variables $z_{1}, z_{2}, z_{3}$ and $z_{4}$ are the coordinates of the four point conformal block in the complex plane. We denote this IRF model as $\operatorname{IRF}(\mathcal{O}, h, v)$.

We find it convenient to define the face transfer matrix as the operator $X_{i}$,

$$
\left\langle a_{1}, a_{2}, \ldots, a_{n}\left|X_{i}(u)\right| a_{1}^{\prime}, a_{2}^{\prime}, \ldots, a_{n}^{\prime}\right\rangle=\left[\prod_{j \neq i} \delta_{a_{j}, a_{j}^{\prime}}\right] \omega\left(\begin{array}{cc}
a_{i-1} & a_{i} \\
a_{i}^{\prime} & a_{i+1}
\end{array} \mid u\right) .
$$

Our purpose is to introduce a solution of the Yang Baxter equation (YBE),

$$
X_{i}(u) X_{i+1}(u+v) X_{i}(v)=X_{i+1}(v) X_{i}(u+v) X_{i+1}(u),
$$

which ensures that the transfer matrices for different spectral parameters, $u$, commute. 
The fusion rules of the primary fields $h$ and $v$ enter into the conformal data:

$$
[h] \times[v]=\sum_{i=0}^{n-1} \psi_{i},
$$

where $[h]$ and $[v]$ are some primary fields in the CFT $\mathcal{O}$. The product is according to the OPE (fusion rules) and $n$ is the number of conformal blocks (for shortness blocks). The eigenvalues of the braiding matrix are given by,

$$
\lambda_{i}=\epsilon_{i} e^{i \pi\left(\Delta_{h}+\Delta_{v}-\Delta_{i}\right)},
$$

where $\epsilon_{i}= \pm 1$, according to whether the product is symmetric or antisymmetric. We shall assume that $h=v$ and $h$ is real, $\psi_{0}=1$, the unit field, and that $\epsilon_{i}=(-1)^{i}$.

Denote by $X_{i}$ the limit of the Brading matrix as $u \rightarrow i \infty$ (up to a factor). We then see that $X_{i}$ obeys an $n$th order polynomial equation,

$$
\prod_{p=0}^{n-1}\left(X_{i}-\lambda_{p}\right)=0
$$

We define the projector operators by,

$$
P_{i}^{a}=\prod_{p \neq a}\left[\frac{X_{i}-\lambda_{p}}{\lambda_{a}-\lambda_{p}}\right] .
$$

The projection operators obey the relations,

$$
\sum_{a=0}^{n-1} P_{i}^{a}=1, \quad P_{i}^{a} P_{i}^{b}=\delta_{a, b} P_{i}^{b}, \quad \sum_{a=0}^{n-1} \lambda_{a} P_{i}^{a}=X_{i} .
$$

In ref. [3], a conjecture for the trigonometric solution of the YBE was introduced. To describe it, we define parameters

$$
\zeta_{i}=\pi\left(\Delta_{i+1}-\Delta_{i}\right) / 2,
$$

where $\Delta_{i}$ is the dimension of $\psi_{i}$. The trigonometric solution of the Yang Baxter equation is then,

$$
X_{i}(u)=\sum_{a=0}^{n-1} f_{a}(u) P_{i}^{a}
$$

where the functions $f_{a}(u)$ are

$$
f_{a}(u)=\left[\prod_{r=1}^{a} \sin \left(\zeta_{r-1}-u\right)\right]\left[\prod_{r=a+1}^{n-1} \sin \left(\zeta_{r-1}+u\right)\right] /\left[\prod_{r=1}^{n-1} \sin \left(\zeta_{r-1}\right)\right] .
$$

For our purposes the following relations will be relevant. First, the Boltzmann weights obey crossing symmetry:

$$
\omega\left(\begin{array}{ll}
a & b \\
c & d
\end{array} \mid \lambda-u\right)=\left[\frac{G_{b} G_{c}}{G_{a} G_{d}}\right]^{1 / 2} \omega\left(\begin{array}{ll}
c & a \\
d & b
\end{array} \mid u\right)
$$


where $G_{a}$ is the crossing multiplier and $\lambda=\zeta_{0}$ is the crossing parameter.

Another relation is the inversion relation for the transfer matrices:

$$
X_{i}(u) X_{i}(-u)=\rho(u) \rho(-u) 1_{i}
$$

where

$$
\rho(u)=\prod_{r=1}^{n-1} \frac{\sin \left(\zeta_{r-1}-u\right)}{\sin \left(\zeta_{r-1}\right)} .
$$

\section{Birman-Murakami-Wenzl algebra}

Our aim is to connect the solvable IRF model with Birman-Murakami-Wenzl algebra [6, 7]. There are two generators of the algebra, $G_{i}$ and $E_{i}$. The relations are,

$$
\begin{aligned}
G_{i} G_{j} & =G_{j} G_{i} \text { if }|i-j| \geq 2, & & \\
G_{i} G_{i+1} G_{i} & =G_{i+1} G_{i} G_{i+1}, & & E_{i} E_{i \pm 1} E_{i}=E_{i}, \\
G_{i}-G_{i}^{-1} & =m\left(1-E_{i}\right), & & \\
G_{i \pm 1} G_{i} E_{i \pm 1} & =E_{i} G_{i \pm 1} G_{i}=E_{i} E_{i \pm 1}, & G_{i \pm 1} E_{i} G_{i \pm 1} & =G_{i}^{-1} E_{i \pm 1} G_{i}^{-1}, \\
G_{i \pm 1} E_{i} E_{i \pm 1} & =G_{i}^{-1} E_{i \pm 1}, & E_{i \pm 1} E_{i} G_{i \pm 1} & =E_{i \pm 1} G_{i}^{-1}, \\
G_{i} E_{i} & =E_{i} G_{i}=l^{-1} E_{i}, & E_{i} G_{i \pm 1} E_{i} & =l E_{i} .
\end{aligned}
$$

These relations imply the additional relations,

$$
E_{i} E_{j}=E_{j} E_{i} \text { if }|i-j| \geq 2, \quad\left(E_{i}\right)^{2}=b E_{i},
$$

where here $b=\left(l-l^{-1}\right) / m+1$. Here $l$ and $m$ are the two parameters of the algebra.

Assume now the three block case, $n=3$. We connect out solvable IRF lattice model by defining

$$
\begin{aligned}
G_{i} & =4 \sin (\lambda) \sin (\mu) e^{-i \lambda} X_{i}, \\
G_{i}^{-1} & =4 \sin (\lambda) \sin (\mu) e^{i \lambda} X_{i}^{t},
\end{aligned}
$$

where

$$
X_{i}^{t}=\lim _{u \rightarrow-i \infty} e^{-2 i u} X_{i}(u)
$$

and the normalization is fixed so that $G_{i} G_{i}^{-1}=1_{i}$ from the inversion relation, eq. (2.13). The phase is arbitrary, and is fixed to be compatible with the BMW algebra. We also define $E_{i}=X_{i}(\lambda)$.

We propose the following Baxterization of the BMW algebra,

$$
U_{i}(u)=1-\frac{i \sin (u)}{2 \sin (\lambda) \sin (\mu)}\left[e^{-i(u-\lambda)} G_{i}-e^{i(u-\lambda)} G_{i}^{-1}\right],
$$

where we identify $\lambda=\zeta_{0}$ and $\mu=\zeta_{1}$. 
For three blocks, the face transfer matrix $X_{i}(u)$, eq. (2.10), assumes the form,

$$
\begin{aligned}
X_{i}(u)= & {\left[P_{i}^{0} \sin \left(\zeta_{0}+u\right) \sin \left(\zeta_{1}+u\right)+P_{i}^{1} \sin \left(\zeta_{0}-u\right) \sin \left(\zeta_{1}+u\right)\right.} \\
& \left.+P_{i}^{2} \sin \left(\zeta_{0}-u\right) \sin \left(\zeta_{1}-u\right)\right] /\left[\sin \left(\zeta_{0}\right) \sin \left(\zeta_{1}\right)\right]
\end{aligned}
$$

We can then see that with this definition, the Baxterized BMW algebra, $U_{i}(u)$, eq. (3.5), and the face transfer matrix, are identical:

$$
U_{i}(u)=X_{i}(u)
$$

We also identify

$$
E_{i}=U_{i}(\lambda)=E_{i}(\lambda)
$$

Several relations are evident from the definitions of $X_{i}(u)$ and $U_{i}(u)$. First, from the crossing symmetry, eq. (2.12), we find that

$$
E\left(\begin{array}{ll}
a & b \\
c & d
\end{array}\right)=\left(\frac{G_{b} G_{c}}{G_{a} G_{d}}\right)^{1 / 2} \delta_{a, d}, \text { and } E_{i} E_{i \pm 1} E_{i}=E_{i} .
$$

From the definition of $E_{i}$ in terms of projection operators, we find,

$$
E_{i}^{2}=b E_{i}, \quad \text { where } b=\frac{\sin (2 \lambda) \sin (\mu+\lambda)}{\sin (\lambda) \sin (\mu)} .
$$

Thus $E_{i}$ obeys the Temperley-Lieb algebra. It is noteworthy that for any number of blocks, the Temperley-Lieb algebra is obeyed with

$$
b=\prod_{r=0}^{n-2} \frac{\sin \left(\lambda+\zeta_{r}\right)}{\sin \left(\zeta_{r}\right)} .
$$

Another relation that is evident is the braiding relation,

$$
\begin{aligned}
G_{i} G_{i+1} G_{i} & =G_{i+1} G_{i} G_{i+1}, \\
G_{i} G_{j} & =G_{j} G_{i} \text { if }|i-j| \geq 2 .
\end{aligned}
$$

From the definition of $E_{i}$ we find the skein relation,

$$
G_{i}-G_{i}^{-1}=m\left(1-E_{i}\right),
$$

where we identify

$$
m=-2 i \sin (\mu),
$$

as one of the parameters of the BMW algebra.

Another relation, which is evident from the definition of the face transfer matrix, $X_{i}(u)$, eq. (3.6), is

$$
G_{i} E_{i}=E_{i} G_{i}=l^{-1} E_{i},
$$

where the parameter $l$ is given by,

$$
l=-e^{i(2 \lambda+\mu)},
$$


which is the second parameter of the BMW algebra. We note, in passing, that this relation, eq. (3.14) is obeyed by any number of blocks, greater than two, with some value of $l$.

One can easily calculate

$$
b=\left(l-l^{-1}\right) / m+1,
$$

which is the relation required by the BMW algebra.

Once establishing these evident relations, which form part of the BMW algebra, we wish to prove that the face transfer matrix, $X_{i}(u)$, obeys the Yang Baxter equation if and only if $G_{i}$ and $E_{i}$ obey the relations of the BMW algebra.

For this purpose, we convert the Yang-Baxter equation to a set of algebraic equations obeyed by $G_{i}$ and $E_{i}$. We do this by inserting $X_{i}(u)$, eq. (3.6), into the YBE, eq. (2.3). We then expand the YBE in terms of $G_{i}, E_{i}$ and $G_{i}^{-1}$, in powers of $e^{i u}$ and $e^{i v}$. We get 19 equations and solve them in terms of the 'basis' elements which is $B_{i} B_{i+1} B_{i}$, where $B_{i}$ is either $G_{i}, E_{i}$ or $G_{i}^{-1}$. We get from this 19 equations which are listed in appendix A.

We wish to show that these equations hold, if and only if, the BMW algebra holds. There are 12 equations which contain a single term only. For example, eq. (A.10) is just the braiding relation, eq. (3.11), which we know that it holds. Similarly, eqs. (A.1)-(A.4), (A.9), are the same braiding relation, where we multiply by $G_{i}$ or $G_{i+1}$ from the left and right. Thus, these equations are all equivalent to the braiding relation, eq. (3.11).

The rest of the one-term equations are all equivalent to one equation. In particular, eqs. (A.11), (A.19) are directly seen to be the BMW relation,

$$
E_{i} G_{i \pm 1} G_{i}=G_{i \pm 1} G_{i} E_{i \pm 1}
$$

The rest of the relations, eqs. (A.4), (A.6), (A.11), (A.12), (A.17) are then seen to be equivalent to this relation, eq. (3.17), by multiplying the l.h.s. and the r.h.s. by the algebra elements $G_{i}$ or $G_{i+1}$.

We get now to the 7 'composite' relations (that is having more than one term). These vary in complexity. First consider the relation (A.5). Using the skein relation, eq. (3.12), we substitute in this relation $G_{j}^{-1} \rightarrow-m+m E_{j}+G_{j}$, where $j=i$ or $i+1$. Then, the relation (A.5) becomes,

$$
0=\left(m+1 / s_{2}-s_{2}\right)\left(-E_{i} E_{i+1} E_{i}+E_{i+1} E_{i} E_{i+1}\right) .
$$

Now, since $m=-2 i \sin \left(\zeta_{1}\right)=s_{2}-1 / s_{2}$, this equation is seen to hold. The relation (A.14) is very similar and is shown to hold in the same way.

Consider now the relation eq. (A.7). Again we substitute $G_{j}^{-1} \rightarrow-m+m E_{j}+G_{j}$ for $j=i$ or $i+1$. We find using the relation $E_{i} E_{i \pm 1} E_{i}=E_{i}$ (the Temperley Lieb relation) that this equation is zero, if and only if,

$$
E_{i} G_{i \pm 1} E_{i}=l E_{i}
$$

This proves one direction of our assertion. Namely, that if the Yang Baxter equation holds then the BMW algebra follows. This is because the BMW algebra is generated by precisely the relations we found $[6,7]$. These are: 
1) The skein relation: $G_{i}-G_{i}^{-1}=m\left(1-E_{i}\right)$.

2) Braid relations: $G_{i} G_{j}=G_{j} G_{i}$ if $|i-j| \geq 2$, and $G_{i} G_{i+1} G_{i}=G_{i+1} G_{i} G_{i+1}$.

3) Tangle relations: $E_{i} E_{i \pm 1} E_{i}=E_{i}$ and $G_{i \pm 1} G_{i} E_{i \pm 1}=E_{i} G_{i \pm 1} G_{i}$.

4) Delooping relations: $G_{i} E_{i}=E_{i} G_{i}=l^{-1} E_{i}$ and $E_{i} G_{i \pm 1} E_{i}=l E_{i}$.

Precisely, these relations we found to hold and thus we proved that the BirmanMurakami-Wenzl algebra holds if the Yang Baxter equation holds.

The rest of the relations can be seen (with some effort) to hold if the BMW algebra holds. The calculations are rather involved and we omit them. This proves that the YBE holds if the BMW algebra is obeyed. We arrive at the conclusion that the Yang Baxter equation is fulfilled, if and only if, the Birman-Murakami-Wenzl algebra is obeyed. Thus, we proved that any three block integrable lattice model obeys the BMW algebra and it is integrable if the BMW algebra holds.

\section{Four blocks lattice IRF models}

We turn now to the four block case. The algebra that governs this models is termed 4-CB (4 Conformal Braiding) algebra. We do not know all the relations of the 4-CB algebra yet. However, the relations that we know are enough to span the algebra.

We denote by BMW' the BMW as described earlier, eq. (3.1), along with the Temperley Lieb algebra, eq. (3.2) (with a different coefficient $b$ ), with all the relations, except, the skein relation. Our first claim is that this algebra $\mathrm{BMW}^{\prime}$, is a sub-algebra of the 4-CB algebra. In other words, the BMW algebra is obeyed, except, obviously, the skein relation which is different. The $\mathrm{BMW}^{\prime}$ algebra is generated by $G_{i}, G_{i}^{-1}$ and $E_{i}$, which are defined below. In fact, we conjecture that the $\mathrm{BMW}^{\prime}$ algebra is obeyed by any number of blocks greater than two, or, it is a sub-algebra of the $n$-CB algebra for $n \geq 3$, again generated by $E_{i}, G_{i}$ and $G_{i}^{-1}$, which are defined similarly. This in analogy to the Temperley-Lieb algebra which, as we proved, eq. (3.9), (3.10), holds for any theory with $n \geq 2$ blocks, generated by $E_{i}$.

As before, we define the 4 -CB algebra as the algebra generated by $G_{i}$ and $E_{i}$ where

$$
\begin{aligned}
G_{i} & =8\left[\prod_{r=0}^{2} \sin \left(\zeta_{r}\right)\right] e^{-3 i \lambda / 2} X_{i}, \\
G_{i}^{-1} & =8\left[\prod_{r=0}^{2} \sin \left(\zeta_{r}\right)\right] e^{3 i \lambda / 2} X_{i}^{t},
\end{aligned}
$$

where $\zeta_{r}$ were defined in eq. (2.9), $\lambda=\zeta_{0}$ is the crossing multiplier, and

$$
X_{i}=\lim _{u \rightarrow i \infty} e^{3 i u} X_{i}(u), \quad X_{i}^{t}=\lim _{u \rightarrow-i \infty} e^{-3 i u} X_{i}(u)
$$

Again

$$
E_{i}=X_{i}(\lambda)
$$


The factor in equation (4.1) is demanded by the inversion relation, eq. (2.13), used to ensure that $G_{i} G_{i}^{-1}=1_{i}$. The phase in the definition of $G_{i}$ is arbitrary, and is set to ensure the relations of the $\mathrm{BMW}^{\prime}$ algebra, as is seen below.

Let us consider the relations of the 4-CB algebra that we already know. First, we have the Temperley-Lieb algebra for $E_{i}$ which is proved to be obeyed, eq. (3.9),

$$
E_{i} E_{i \pm 1} E_{i}=E_{i}
$$

In addition, we have,

$$
E_{i}^{2}=b E_{i}, \quad \text { where } b=\prod_{r=0}^{2} \frac{\sin \left(\lambda+\zeta_{r}\right)}{\sin \left(\zeta_{r}\right)} .
$$

This we see by substituting $u=\lambda$ in eq. (2.10) and using $\left(P_{i}^{0}\right)^{2}=P_{i}^{0}$.

The next relations are

$$
G_{i} E_{i}=E_{i} G_{i}=l^{-1} E_{i} .
$$

These relations are verified by substituting $G_{i}$ from the definition, eq. (4.1), and using $P_{i}^{a} P_{i}^{0}=P_{i}^{0} P_{i}^{a}=\delta_{a, 0} P_{i}^{0}$. We find for $l$ the value,

$$
l=i e^{i\left(3 \lambda / 2+\zeta_{0}+\zeta_{1}+\zeta_{2}\right)} .
$$

Now, we know the braiding relations for $G_{i}$ :

$$
G_{i} G_{j}=G_{j} G_{i} \text { if }|i-j| \geq 2, \quad G_{i} G_{i+1} G_{i}=G_{i+1} G_{i} G_{i+1} .
$$

The obvious relation not in $\mathrm{BMW}^{\prime}$ is the skein relation which is,

$$
G_{i}^{2}=\alpha+\beta E_{i}+\gamma G_{i}+\delta G_{i}^{-1}
$$

where $\alpha, \beta, \gamma, \delta$ are constants, which depend on $\zeta_{r}, r=0,1,2$. These constants are given in appendix C, eq. (C.8).

From the skein relation, eq. (4.9) we find the relation

$$
G_{i \pm 1} G_{i} E_{i \pm 1}=E_{i} G_{i \pm 1} G_{i}
$$

which follows from the $\mathrm{BMW}^{\prime}$ algebra along with the skein relation.

We also find the relation,

$$
E_{i} G_{i \pm 1}^{2} E_{i}=\kappa E_{i}
$$

which again follows from the BMW' algebra along with the skein relation, by substituting the value of $G_{i}^{2}$. The coefficient $\kappa$, which depends on $\zeta_{i}$ is determined from this and is

$$
\kappa=\alpha b+\beta+\gamma l+\delta l^{-1} .
$$

For the $\mathrm{SU}(2)$ fused model (section 5 ) we have $\kappa=1$, but this is not true for the general four block model. 
In fact, the skein relation allows us to express $G_{i}^{2}$ in terms of $G_{i}, E_{i}$ and $G_{i}^{-1}$. Since, as was argued above, the latter satisfy the $\mathrm{BMW}^{\prime}$ algebra, these relations are exactly enough to span the entire 4-CB algebra, i.e., the four block algebra. The additional relations, involving $G_{i}^{2}$, which are only partially known, will be important as relations of the five block algebra.

Another consequence is that we can define a knot polynomial relation from the 4$\mathrm{CB}$ algebra. The knot polynomials are defined from words in the braid group enveloping algebra, representing the particular knot, see e.g. [9]. Thus, this relations are exactly enough to reduce every such word down to unity. This defines a new knot invariant. The details are given in appendix D.

\section{The fused $3 \times 3 \mathrm{SU}(2)$ model}

Let us give now a concrete example of a four block IRF lattice model. This is the model $\operatorname{IRF}\left(\mathrm{SU}(2)_{k},[3],[3]\right)$. Namely, the conformal field theory $\mathcal{O}$ is $\mathrm{SU}(2)_{k}$ and the fields $h=v=$ [3], i.e., the isospin $3 / 2$ representation. We denote by $l$ the isospin of the representation and $l=0,1,2, \ldots, k$.

The fields appearing in the fusion product of $h \times v$ are

$$
[3] \times[3]=[0]+[2]+[4]+[6] .
$$

So, this is a four block theory.

The dimension formula for $\mathrm{SU}(2)_{k}$ for the representation $[l]$ is

$$
\Delta_{l}=\frac{l(l+2)}{4(k+2)}
$$

The parameters $\zeta_{i}$ are given by

$$
\begin{aligned}
& \zeta_{0}=\lambda=\frac{\pi}{2}\left(\Delta_{2}-\Delta_{0}\right)=\frac{\pi}{k+2} \\
& \zeta_{1}=\frac{\pi}{2}\left(\Delta_{4}-\Delta_{2}\right)=2 \lambda
\end{aligned}
$$

and

$$
\zeta_{2}=\frac{\pi}{2}\left(\Delta_{6}-\Delta_{4}\right)=3 \lambda
$$

The Boltzmann weights of this model are listed in appendix B. We checked that all the relations described by eq. (3.1), (3.2), except for the skein relation, are obeyed, numerically. In particular, we verified the BMW' algebra described there. The parameters $l$ and $b$ are seen to be,

$$
l=i e^{i\left(3 \lambda / 2+\zeta_{0}+\zeta_{1}+\zeta_{2}\right)}=i e^{i(15 \lambda / 2)}, \quad b=\frac{\sin (4 \lambda)}{\sin (\lambda)},
$$

in accordance with eqs. (4.5), (4.7). We checked the algebra at levels $k=8,10,11$. We find a complete agreement with the BMW' algebra, as described in section 4 . We were not able to check this algebra for general $k$ due to the complexity of the calculation.

We also checked that the algebraic relations coming from expanding the YBE are all obeyed for this four block model. The details are given in appendix C. 


\section{Discussion}

In this paper, and the previous one [5], we investigated the algebraic structure of solvable lattice models. The related algebras were termed $n$-CB algebras, where $n$ is the number of blocks. We found that the 3-CB algebra is the Birman-Murakami-Wenzl algebra for any three block theory. For the 4-CB algebra, we argued that it is generated by the BMW algebra with a different skein relation. Clearly, more study is needed. In particular, deciphering the general $n$-CB algebra, for any $n$, is a major challenge, left to future work. Our present results indicate that the BMW algebra is a sub-algebra of the $n$-CB algebra for any $n$, with different skein relations.

In physics, the knowledge of the algebraic structure of solvable lattice models would contribute to the study of solvable lattice models, conformal field theory and integrable soliton systems.

In mathematics, the studies of such models is important to knot theory, yielding new knot invariants. In particular, as we indicated the 4-CB algebra gives a new knot invariant which was described in appendix D. The IRF lattice models are also important in combinatorics, yielding new Rogers-Ramanujan type identities. For examples, see [1].

\section{Acknowledgments}

We are extremely indebted to Ida Deichaite for her encouragement and impetus, without which this paper would probably not have been written. We are grateful for discussions with B. Le Floch, H. Wenzl and J.B. Zuber.

\section{A The relations of the three block YBE algebra}

We list the 19 relations obtained by expanding the YBE in powers of $e^{i u}$ and $e^{i v}$. We denote by $a_{i, j, k}[r, s, t]$ the element of the algebra $a_{i}[r] a_{j}[s] a_{k}[t]$, where $a_{i}[r]$ is $G_{r}, G_{r}^{-1}$ or $E_{r}$ according to whether $i=1,2,3$, respectively. We define here,

$$
s_{1}=e^{-i \zeta_{0}}, \quad s_{2}=e^{-i \zeta_{1}} .
$$

The 19 relations of the three block model are then calculated to be given as follows,

$$
\begin{aligned}
a_{2,1,1}(i, i+1, i)= & a_{1,1,2}(i+1, i, i+1), \\
a_{2,2,1}(i, i+1, i)= & a_{1,2,2}(i+1, i, i+1), \\
a_{2,2,2}(i, i+1, i)= & a_{2,2,2}(i+1, i, i+1), \\
a_{2,2,3}(i, i+1, i)= & a_{3,2,2}(i+1, i, i+1), \\
a_{3,3,1}(i, i+1, i)= & \left(\frac{1}{s_{2}}-s_{2}\right) a_{3,3,3}(i, i+1, i) \\
& +\left(s_{2}-\frac{1}{s_{2}}\right) a_{3,3,3}(i+1, i, i+1) \\
& +a_{1,3,3}(i+1, i, i+1)-a_{2,3,3}(i+1, i, i+1) \\
& +a_{3,3,2}(i, i+1, i),
\end{aligned}
$$




$$
\begin{aligned}
& a_{3,2,2}(i, i+1, i)=a_{2,2,3}(i+1, i, i+1), \\
& a_{3,1,3}(i, i+1, i)=\frac{a_{3,2,3}(i, i+1, i)}{s_{1}^{4}}-\frac{a_{3,2,3}(i+1, i, i+1)}{s_{1}} \\
& +\frac{\left(s_{2}^{2}-1\right) a_{3,3,3}(i, i+1, i)}{s_{1}^{2} s_{2}} \\
& +\frac{\left(1-s_{2}^{2}\right) a_{3,3,3}(i+1, i, i+1)}{s_{1}^{2} s_{2}}+a_{3,1,3}(i+1, i, i+1), \\
& a_{1,2,2}(i, i+1, i)=a_{2,2,1}(i+1, i, i+1), \\
& a_{1,1,2}(i, i+1, i)=a_{2,1,1}(i+1, i, i+1), \\
& a_{1,1,1}(i, i+1, i)=a_{1,1,1}(i+1, i, i+1) \text {, } \\
& a_{1,1,3}(i, i+1, i)=a_{3,1,1}(i+1, i, i+1) \text {, } \\
& a_{2,3,1}(i, i+1, i)=a_{1,3,2}(i+1, i, i+1) \text {, } \\
& a_{2,1,3}(i, i+1, i)=-\frac{\left(s_{1}^{2}-1\right)\left(s_{2}^{2}-1\right)^{2} a_{3,3,3}(i, i+1, i)}{s_{1}^{2} s_{2}^{2}} \\
& +\frac{\left(s_{1}^{2}-1\right)\left(s_{2}^{2}-1\right)^{2} a_{3,3,3}(i+1, i, i+1)}{s_{1}^{2} s_{2}^{2}} \\
& -\frac{\left(s_{1}^{2}-1\right)\left(s_{2}^{2}-1\right) a_{1,3,3}(i, i+1, i)}{s_{1}^{2} s_{2}} \\
& +\frac{\left(s_{1}^{2}-1\right)\left(s_{2}^{2}-1\right) a_{3,3,1}(i+1, i, i+1)}{s_{1}^{2} s_{2}} \\
& -\frac{a_{1,2,3}(i, i+1, i)}{s_{1}^{2}}+\frac{a_{3,2,1}(i+1, i, i+1)}{s_{1}^{2}} \\
& -\frac{\left(s_{1}^{2}-1\right)\left(s_{2}^{2}-1\right) a_{3,2,3}(i, i+1, i)}{s_{1}^{4} s_{2}} \\
& +\frac{\left(s_{1}^{2}-1\right)\left(s_{2}^{2}-1\right) a_{3,2,3}(i+1, i, i+1)}{s_{1}^{4} s_{2}} \\
& +a_{3,1,2}(i+1, i, i+1), \\
& a_{2,3,3}(i, i+1, i)=\left(s_{2}-\frac{1}{s_{2}}\right) a_{3,3,3}(i, i+1, i) \\
& +\left(\frac{1}{s_{2}}-s_{2}\right) a_{3,3,3}(i+1, i, i+1) \\
& +a_{1,3,3}(i, i+1, i)-a_{3,3,1}(i+1, i, i+1)+a_{3,3,2}(i+1, i, i+1) \text {, } \\
& a_{3,1,2}(i, i+1, i)=\frac{s_{2} a_{1,2,1}(i, i+1, i)}{\left(s_{1}^{2}-1\right)\left(s_{2}^{2}-1\right)}-\frac{s_{2} a_{1,2,1}(i+1, i, i+1)}{\left(s_{1}^{2}-1\right)\left(s_{2}^{2}-1\right)} \\
& -\frac{s_{2} a_{2,1,2}(i, i+1, i)}{\left(s_{1}^{2}-1\right)\left(s_{2}^{2}-1\right)}+\frac{s_{2} a_{2,1,2}(i+1, i, i+1)}{\left(s_{1}^{2}-1\right)\left(s_{2}^{2}-1\right)} \\
& +\frac{\left(s_{1}^{2}-1\right)\left(s_{2}^{2}-1\right) a_{2,3,3}(i+1, i, i+1)}{s_{1}^{2} s_{2}} \\
& -\frac{\left(s_{1}^{2}-1\right)\left(s_{2}^{2}-1\right) a_{3,3,2}(i, i+1, i)}{s_{1}^{2} s_{2}}
\end{aligned}
$$




$$
\begin{aligned}
&+\frac{a_{1,2,3}(i, i+1, i)}{s_{1}^{2}}-\frac{a_{2,3,2}(i, i+1, i)}{s_{1}^{2}} \\
&+\frac{a_{2,3,2}(i+1, i, i+1)}{s_{1}^{2}}-\frac{a_{3,2,1}(i+1, i, i+1)}{s_{1}^{2}} \\
&+a_{2,1,3}(i+1, i, i+1) \\
& a_{1,3,1}(i, i+1, i)=-\frac{\left(s_{1}^{2}-1\right)\left(s_{2}^{2}-1\right) a_{1,3,3}(i, i+1, i)}{s_{1}^{2} s_{2}} \\
&+\frac{\left(s_{1}^{2}-1\right)\left(s_{2}^{2}-1\right) a_{2,3,3}(i+1, i, i+1)}{s_{1}^{2} s_{2}} \\
&+\frac{\left(s_{1}^{2}-1\right)\left(s_{2}^{2}-1\right) a_{3,3,1}(i+1, i, i+1)}{s_{1}^{2} s_{2}} \\
&-\frac{\left(s_{1}^{2}-1\right)\left(s_{2}^{2}-1\right) a_{3,3,2}(i, i+1, i)}{s_{1}^{2} s_{2}} \\
&-\frac{a_{2,3,2}(i, i+1, i)}{s_{1}^{2}}+\frac{a_{2,3,2}(i+1, i, i+1)}{s_{1}^{2}}+a_{1,3,1}(i+1, i, i+1), \\
& a_{1,3,2}(i, i+1, i)= a_{2,3,1}(i+1, i, i+1), \\
& a_{3,2,1}(i, i+1, i)=-\frac{s_{1}^{2} s_{2} a_{1,2,1}(i, i+1, i)}{\left(s_{1}^{2}-1\right)\left(s_{2}^{2}-1\right)}+\frac{s_{1}^{2} s_{2} a_{1,2,1}(i+1, i, i+1)}{\left(s_{1}^{2}-1\right)\left(s_{2}^{2}-1\right)} \\
&+\frac{s_{1}^{2} s_{2} a_{2,1,2}(i, i+1, i)}{\left(s_{1}^{2}-1\right)\left(s_{2}^{2}-1\right)}-\frac{s_{1}^{2} s_{2} a_{2,1,2}(i+1, i, i+1)}{\left(s_{1}^{2}-1\right)\left(s_{2}^{2}-1\right)} \\
&-\frac{\left(s_{1}^{2}-1\right)\left(s_{2}^{2}-1\right) a_{3,2,3}(i, i+1, i)}{s_{1}^{2} s_{2}} \\
&+\frac{\left(s_{1}^{2}-1\right)\left(s_{2}^{2}-1\right) a_{3,2,3}(i+1, i, i+1)}{s_{1,1,1}^{2} s_{2}} \\
&-a_{1,2,3}(i, i+1, i)+a_{1,2,3}(i+1, i, i+1)+a_{2,3,2}(i, i+1, i) \\
&-a_{2,3,2}(i+1, i, i+1)+a_{3,2,1}(i+1, i, i+1), \\
& a_{1,1,3}(i+1, i, i+1) .
\end{aligned}
$$

\section{B Weights of the $3 \times 3$ fused model}

These are the weights of the model $\operatorname{IRF}\left(\mathrm{SU}(2)_{k},[3],[3]\right)$. We use the notation,

$$
\lambda=\frac{\pi}{k+2}
$$

and

$$
s[x]=\frac{\sin (x)}{\sin (\lambda)} .
$$

The weights are taken from ref. [13], based on the calculations of ref. [8]. We shifted the fields $a \rightarrow a+1$ so the weights range over $a=1,2, \ldots, k+1$, namely, $a$ is the dimension of the $\mathrm{SU}(2)$ representation.

$\omega\left(\begin{array}{cc}a \pm 3 & a \\ a & a \pm 3\end{array} \mid u\right)=\frac{s((a \pm 1) \lambda \mp u) s((a \pm 2) \lambda \mp u) s((a \pm 3) \lambda \mp u)}{s((a \pm 1) \lambda) s((a \pm 2) \lambda) s((a \pm 3) \lambda)}$ 


$$
\begin{aligned}
& \omega\left(\begin{array}{cc}
a \mp 3 & a \\
a & a \pm 3
\end{array} \mid u\right)=\frac{s(\lambda-u) s(2 \lambda-u) s(3 \lambda-u)}{s(2 \lambda) s(3 \lambda)} \\
& \omega\left(\begin{array}{cc}
a \pm 1 & a \\
a & a \pm 3
\end{array} \mid u\right)=\omega\left(\begin{array}{cc}
a \pm 3 & a \\
a & a \pm 1
\end{array} \mid u\right)=\frac{s(\lambda-u) s((a \pm 1) \lambda \mp u) s((a \pm 2) \lambda \mp u)}{s((a \pm 1) \lambda) s((a \pm 2) \lambda)} \\
& \omega\left(\begin{array}{cc}
a \mp 1 & a \\
a & a \pm 3
\end{array} \mid u\right)=\omega\left(\begin{array}{cc}
a \pm 3 & a \\
a & a \mp 1
\end{array} \mid u\right)=\frac{s(\lambda-u) s(2 \lambda-u) s((a \pm 1) \lambda \mp u)}{s(2 \lambda) s((a \pm 1) \lambda)} \\
& \omega\left(\begin{array}{cc}
a \pm 1 & a \\
a & a \pm 1
\end{array} \mid u\right)=\frac{s((a \pm 1) \lambda \mp u) s((a \pm 1) \lambda \pm u) s((a \pm 2) \lambda \mp u)}{s((a \pm 1) \lambda)^{2} s((a \pm 2) \lambda)} \\
& -\frac{s(2 \lambda) s((a-2) \lambda) s((a+2) \lambda) s(\lambda-u) s(u) s((a \pm 1) \lambda \mp u)}{s(3 \lambda) s((a \mp 1) \lambda) s((a \pm 1) \lambda)^{2}} \\
& \omega\left(\begin{array}{cc}
a \mp 1 & a \\
a & a \pm 1
\end{array} \mid u\right)=\frac{s(2 \lambda)^{2} s((a \mp 2) \lambda) s(\lambda-u) s(a \lambda \pm u) s((a \pm 1) \lambda \mp u)}{s(3 \lambda) s((a \mp 1) \lambda)^{2} s((a \pm 1) \lambda)} \\
& -\frac{s((a \mp 3) \lambda) s((a \pm 1) \lambda) s(2 \lambda-u) s(\lambda-u) s(\lambda+u)}{s(2 \lambda) s(3 \lambda) s((a \mp 1) \lambda)^{2}} \\
& \omega\left(\begin{array}{cc}
a \pm 3 & a \\
a \pm 6 & a \pm 3
\end{array} \mid u\right)=-\frac{s((a \pm 4) \lambda) s((a \pm 5) \lambda) s((a \pm 6) \lambda) s(u) s(\lambda+u) s(2 \lambda+u)}{s(2 \lambda) s(3 \lambda) s((a \pm 1) \lambda) s((a \pm 2) \lambda) s((a \pm 3) \lambda)} \\
& \omega\left(\begin{array}{cc}
a \pm 3 & a \\
a \pm 4 & a \pm 3
\end{array} \mid u\right)=\frac{s((a \pm 4) \lambda) s((a \pm 5) \lambda) s(u) s(\lambda+u) s((a \pm 3) \lambda \mp u)}{s(2 \lambda) s(3 \lambda) s((a \pm 1) \lambda) s((a \pm 2) \lambda) s((a \pm 3) \lambda)} \\
& \omega\left(\begin{array}{cc}
a \pm 1 & a \\
a \pm 4 & a \pm 3
\end{array} \mid u\right)=\omega\left(\begin{array}{cc}
a \pm 3 & a \\
a \pm 4 & a \pm 1
\end{array} \mid u\right)=-\frac{s((a \pm 4) \lambda) s((a \pm 5) \lambda) s(u) s(u-\lambda) s(\lambda+u)}{s(2 \lambda) s(3 \lambda) s((a \pm 1) \lambda) s((a \pm 2) \lambda)} \\
& \omega\left(\begin{array}{cc}
a \pm 3 & a \\
a \pm 2 & a \pm 3
\end{array} \mid u\right)=-\frac{s((a \pm 4) \lambda) s(u) s((a \pm 2) \lambda \mp u) s((a \pm 3) \lambda \mp u)}{s(3 \lambda) s((a \pm 1) \lambda) s((a \pm 2) \lambda) s((a \pm 3) \lambda)} \\
& \omega\left(\begin{array}{cc}
a \pm 1 & a \\
a \pm 2 & a \pm 3
\end{array} \mid u\right)=\omega\left(\begin{array}{cc}
a \pm 3 & a \\
a \pm 2 & a \pm 1
\end{array} \mid u\right)=\frac{s((a \pm 4) \lambda) s(u) s(u-\lambda) s((a \pm 2) \lambda \mp u)}{s(3 \lambda) s((a \pm 1) \lambda) s((a \pm 2) \lambda)} \\
& \omega\left(\begin{array}{cc}
a \mp 1 & a \\
a \pm 2 & a \pm 3
\end{array} \mid u\right)=\omega\left(\begin{array}{cc}
a \pm 3 & a \\
a \pm 2 & a \mp 1
\end{array} \mid u\right)=-\frac{s((a \pm 4) \lambda) s(2 \lambda-u) s(\lambda-u) s(u)}{s(2 \lambda) s(3 \lambda) s((a \pm 1) \lambda)} \\
& \omega\left(\begin{array}{lc}
a \pm 1 & a \\
a \pm 4 & a \pm 1
\end{array} \mid u\right)=\frac{s(3 \lambda) s((a \pm 3) \lambda)(s(a \pm 4) \lambda) s(u) s(u+\lambda) s((a \pm 1) \lambda \pm u)}{s(2 \lambda) s((a-1) \lambda) s((a+1) \lambda) s((a \pm 2) \lambda)} \\
& \omega\left(\begin{array}{lc}
a \pm 1 & a \\
a \pm 2 & a \pm 1
\end{array} \mid u\right)=-\frac{s(a \lambda) s((a \pm 3) \lambda) s((a \pm 4) \lambda) s(u)^{2} s(u-\lambda)}{s(2 \lambda) s(3 \lambda) s((a \pm 1) \lambda)^{2} s((a \pm 2) \lambda)} \\
& -\frac{s((a \pm 3) \lambda) s(u) s(a \lambda \pm u) s((a \pm 1) \lambda \mp u)}{s((a \mp 1) \lambda) s((a \pm 1) \lambda)^{2}} \\
& \omega\left(\begin{array}{cc}
a \mp 1 & a \\
a \pm 2 & a \pm 1
\end{array} \mid u\right)=\omega\left(\begin{array}{cc}
a \pm 1 & a \\
a \pm 2 & a \mp 1
\end{array} \mid u\right)=\frac{s((a \pm 3) \lambda) s(u) s(u-\lambda) s(a \lambda \pm u)}{s((a-1) \lambda) s((a+1) \lambda)} \\
& \omega\left(\begin{array}{cc}
a \pm 1 & a \\
a \mp 2 & a \pm 1
\end{array} \mid u\right)=-\frac{s(3 \lambda) s((a \mp 2) \lambda) s(u) s(a \lambda \mp u) s((a \pm 1) \lambda \mp u)}{s((a-1) \lambda) s((a+1) \lambda) s((a \pm 2) \lambda)}
\end{aligned}
$$




\section{The algebraic expansion of the four block YBE}

According to the conjecture in [3] (checked recently for 3-block case in [5]), the trigonometric solution of the YBE, eq. (2.3) is given by eq. (2.10). The generators of the desired algebra are defined to be proportional to the limiting values of $X_{i}$ and $X_{i}^{t}$, arising in the limit $u \rightarrow \pm i \infty$.

Hence, in the four-block case we can identify

$$
\begin{aligned}
G_{i} & =8 e^{\frac{1}{2}(-(3 i)) \zeta_{0}} \sin \left(\zeta_{0}\right) \sin \left(\zeta_{1}\right) \sin \left(\zeta_{2}\right)\left(\lim _{\mathrm{u} \rightarrow i \infty} \exp (3 i \mathrm{u}) X_{i}(\mathrm{u})\right) \\
G_{i}^{-1} & =8 e^{\frac{1}{2}(3 i) \zeta_{0}} \sin \left(\zeta_{0}\right) \sin \left(\zeta_{1}\right) \sin \left(\zeta_{2}\right)\left(\lim _{\mathrm{u} \rightarrow-i \infty} \exp (-3 i \mathrm{u}) X_{i}(\mathrm{u})\right) \\
E_{i} & =X_{i}\left(\zeta_{0}\right)
\end{aligned}
$$

Taking into account the properties of the projectors, eq. (2.8), we can also introduce forth relation for the generator $G_{i}^{2}$.

Explicitly we have

$$
\begin{aligned}
& G_{i}= i e^{-\frac{5}{2} i \zeta_{0}-i \zeta_{1}-i \zeta_{2}}\left(e^{2 i \zeta_{0}} P_{i}^{1}-e^{2 i \zeta_{0}+2 i \zeta_{1}} P_{i}^{2}\right. \\
&\left.+e^{2 i \zeta_{0}+2 i \zeta_{1}+2 i \zeta_{2}} P_{i}^{3}-P_{i}^{0}\right), \\
& G_{i}^{-1}= i e^{\frac{1}{2} i \zeta_{0}-i \zeta_{1}-i \zeta_{2}}\left(e^{2 i \zeta_{0}+2 i \zeta_{1}+2 i \zeta_{2}} P_{i}^{0}\right. \\
&\left.-e^{2 i \zeta_{1}+2 i \zeta_{2}} P_{i}^{1}+e^{2 i \zeta_{2}} P_{i}^{2}-P_{i}^{3}\right), \\
& E_{i}=\frac{e^{-3 i \zeta_{0}}\left(1+e^{2 i \zeta_{0}}\right)\left(-1+e^{i \zeta_{0}+i \zeta_{1}}\right)\left(1+e^{i \zeta_{0}+i \zeta_{1}}\right)}{\left(-1+e^{i \zeta_{1}}\right)\left(1+e^{i \zeta_{1}}\right)\left(-1+e^{i \zeta_{2}}\right)\left(1+e^{i \zeta_{2}}\right)} \\
& \times\left(-1+e^{i \zeta_{0}+i \zeta_{2}}\right)\left(1+e^{i \zeta_{0}+i \zeta_{2}}\right) P_{i}^{0}, \\
& G_{i}^{2}=-e^{-5 i \zeta_{0}-2 i \zeta_{1}-2 i \zeta_{2}} P_{i}^{0}-e^{-i \zeta_{0}-2 i \zeta_{1}-2 i \zeta_{2}} P_{i}^{1} \\
&-e^{-i \zeta_{0}+2 i \zeta_{1}-2 i \zeta_{2}} P_{i}^{2}-e^{-i \zeta_{0}+2 i \zeta_{1}+2 i \zeta_{2}} P_{i}^{3} .
\end{aligned}
$$

That is we have the system of four linear equations, which allows to express four projectors in terms of four generators $G_{i}, G_{i}^{-1}, E_{i}$ and $G_{i}^{2}$. Using these expressions for projectors and eq. (2.10), we get $X_{i}(u)$ expressed in terms of the desired algebra generators.

Finally, we note that the dependence on the spectral parameters $u, v$ in the YBE equation, enters only through the coefficients $f_{a}(u)$. So that The YBE becomes a polynomial equation in the two variables $e^{i u}$ and $e^{i v}$, which is equivalent to the requirement that all the coefficients are equal to zero. This gives a set of three-linear relations for the new algebra generators. 

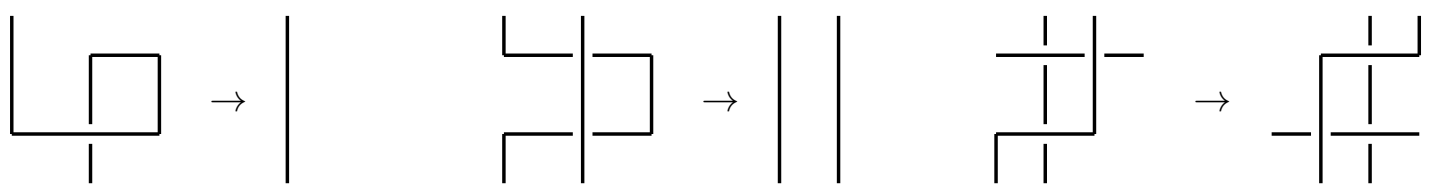

Figure 2. Reidemeister moves.

By using the equation $\sum_{a} P_{i}^{a}=1$ we get the skein relation expressing $G_{i}^{2}$ in terms of $G_{i}, E_{i}$ and $G_{i}^{-1}$. The skein equation is then seen to be,

$$
\begin{aligned}
G_{i}^{2}= & i e^{-\frac{1}{2} i \zeta_{0}-i \zeta_{1}-i \zeta_{2}}\left(1-e^{2 i \zeta_{1}}+e^{2 i \zeta_{1}+2 i \zeta_{2}}\right) G_{i}+i e^{-\frac{3}{2} i \zeta_{0}+i \zeta_{1}-i \zeta_{2}} G_{i}^{-1} \\
& +\frac{e^{-2 i \zeta_{0}-2 i \zeta_{1}-2 i \zeta_{2}}\left(e^{2 i \zeta_{1}}-1\right)\left(1+e^{2 i \zeta_{0}+2 i \zeta_{1}+2 i \zeta_{2}}\right)\left(e^{2 i \zeta_{2}}-1\right)}{\left(e^{2 i \zeta_{0}+2 i \zeta_{2}}-1\right)} E_{i} \\
& -e^{-i \zeta_{0}-2 i \zeta_{2}}\left(1-e^{2 i \zeta_{2}}+e^{2 i \zeta_{1}+2 i \zeta_{2}}\right) .
\end{aligned}
$$

We define the coefficients $\alpha, \beta, \gamma$ and $\delta$ by equating eq. (C.8) to

$$
G_{i}^{2}=\alpha+\beta E_{i}+\gamma G_{i}+\delta G_{i}^{-1} .
$$

\section{New knot invariants}

We define an invariant on a link diagram $K$ as follows,

$$
v(K)=l^{w(K)} L(K),
$$

where $w(K)$ is the writhe of the link $K$ which is defined as the number of left crossings minus the number of right crossings, and $l$ is given by eq. (4.7) and is a parameter.

We define the link function $L(K)$ as follows,

1) $L(0)=1$,

2) $L\left(S_{r}\right)=l^{-1} L(S)$ and $L\left(S_{l}\right)=l L(S)$,

3) $L$ is unchanged under type II, III Reidemeister moves, see figure 2.

Here 0 is the unknot, $S$ is a strand and $S_{r}$ (respectively $S_{l}$ ) is the same strand with a right-handed (respectively left-handed) curl added, as in type I Reidemeister move.

In addition $L$ obeys the skein relations,

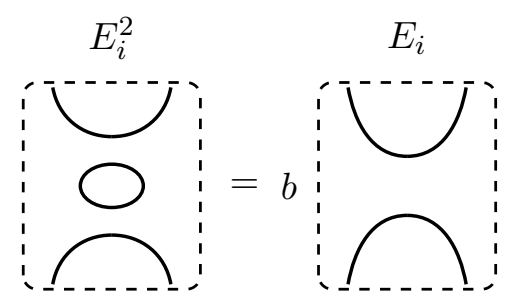


and

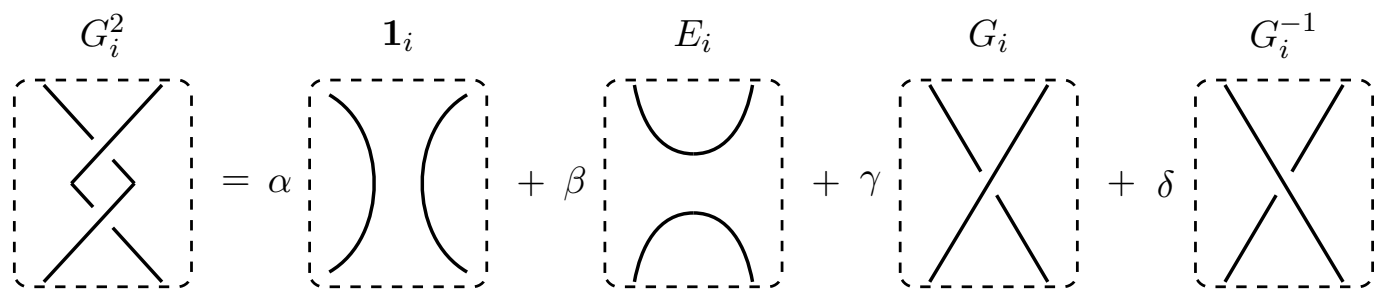

where $\alpha, \beta, \gamma, \delta$ are given by eq. (C.8) and $b$ by eq. (4.5). It also obeys, from the skein relation, eq. (C.8), (C.9),

$$
b=1 / \beta\left(1 / l^{2}-\alpha-\gamma / l-\delta l\right) .
$$

This is a three parameter tangle algebra depending on $\zeta_{0}, \zeta_{1}, \zeta_{2}$. This tangle algebra is isomorphic to the $\mathrm{BMW}^{\prime}$ algebra. The isomorphism is given by,

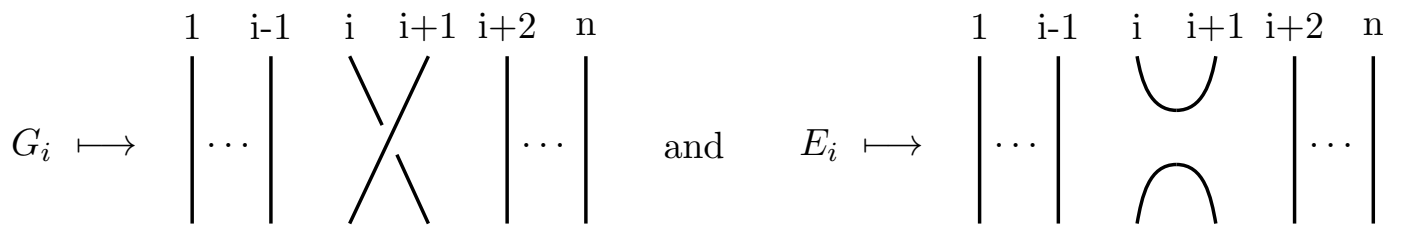

The BMW' algebra ensures invariance under Reidemeister moves and skein relations.

Using this tangle algebra any knot invariant can be calculated. The fact that this $L(K)$ exists and is a regular isotopy invariant follows from the consistency of the BMW' algebra, for which we have explicit representation for some $\zeta_{i}$, which correspond to some solvable lattice model. Thus, $v(K)$ is an invariant (ambient isotopy invariant) of oriented links. For the general values of the parameters, we did not prove the consistency of the $\mathrm{BMW}^{\prime}$ algebra, and this is left to further work.

This defines a three parameter link invariant. It is, in fact, the same invariant defined through the Boltzmann weights in refs. $[2,9]$, which we term the IRF invariant. The advantage of our approach is the following. First, our link invariant can be calculated by the skein relations, unlike the IRF knot invariant where one cannot express $G_{i}^{2}$, for four block theories. Second, our invariant holds for all the values of the parameters $\zeta_{0}, \zeta_{1}, \zeta_{2}$, and is thus a three parameter link invariant, whereas the IRF invariant is special to such values of the parameters appearing in conformal field theory.

The benefit of our three parameter link invariant is that it could be used to distinguish links which cannot be told apart by existing link invariants.

Open Access. This article is distributed under the terms of the Creative Commons Attribution License (CC-BY 4.0), which permits any use, distribution and reproduction in any medium, provided the original author(s) and source are credited.

\section{References}

[1] R.J. Baxter, Exactly solved models in statistical mechanics, Academic Press, London, U.K. (1982) [INSPIRE]. 
[2] M. Wadati, T. Deguchi and Y. Akutsu, Exactly solvable models and knot theory, Phys. Rept. 180 (1989) 247 [inSPIRE].

[3] D. Gepner, Foundations of rational quantum field theory, I, hep-th/9211100 [INSPIRE].

[4] D. Gepner and E. Witten, String theory on group manifolds, Nucl. Phys. B 278 (1986) 493 [INSPIRE].

[5] V. Belavin and D. Gepner, Three blocks solvable lattice models and Birman-Murakami-Wenzl algebra, Nucl. Phys. B 938 (2019) 223 [arXiv:1807.05603] [InSPIRE].

[6] J.S. Birman and H. Wenzl, Braids, link polynomials and a new algebra, Trans. Amer. Math. Soc. 313 (1989) 249.

[7] J. Murakami, The Kauffman polynomial of links and representation theory, Osaka J. Math. 24 (1987) 745 [INSPIRE].

[8] E. Date, M. Jimbo, T. Miwa and M. Okado, Fusion of the eight vertex SOS model, Lett. Math. Phys. 12 (1986) 209 [Erratum ibid. 14 (1987) 97] [INSPIRE].

[9] D. Gepner, On solvable lattice models and knot invariants, hep-th/9305182 [INSPIRE].

[10] W. Galleas and M.J. Martins, R-matrices and spectrum of vertex models based on superalgebras, Nucl. Phys. B 699 (2004) 455 [nlin/0406003] [InSPIRE].

[11] E. Vernier, J.L. Jacobsen and H. Saleur, Non compact conformal field theory and the $a_{2}^{(2)}$ (Izergin-Korepin) model in regime III, J. Phys. A 47 (2014) 285202 [arXiv:1404.4497] [INSPIRE].

[12] G.W. Moore and N. Seiberg, Polynomial equations for rational conformal field theories, Phys. Lett. B 212 (1988) 451 [INSPIRE].

[13] E. Tartaglia and P.A. Pearce, Fused RSOS lattice models as higher-level nonunitary minimal cosets, J. Phys. A 49 (2016) 184002 [arXiv:1509.07576] [inSPIRE]. 